\title{
SNPTB: nucleotide variant identification and annotation in Mycobacterium tuberculosis genomes
}

\author{
Aditi Gupta* \\ Center for Emerging Pathogens, Rutgers New Jersey Medical School, 185 South \\ Orange Avenue, Newark, NJ 07103, USA
}

${ }^{*}$ To whom correspondence should be addressed

\begin{abstract}
Summary: Whole genome sequencing (WGS) has become a mainstay in biomedical research. The continually decreasing cost of sequencing has resulted in a data deluge that underlines the need for easy-to-use bioinformatics pipelines that can mine meaningful information from WGS data. SNPTB is one such pipeline that analyzes WGS data originating from in vitro or clinical samples of Mycobacterium tuberculosis and outputs high-confidence single nucleotide polymorphisms in the bacterial genome. The name of the mutated gene and the functional consequence of the mutation on the gene product is also determined. SNPTB utilizes open source software for WGS data analyses and is written primarily for biologists with minimal computational skills.
\end{abstract}

Availability and implementation: SNPTB is a python package and is available from https://github.com/aditi9783/SNPTB

Contact: ag1349@njms.rutgers.edu

Supplementary information: Tutorial for SNPTB is available at https://github.com/aditi9783/SNPTB/blob/master/docs/SNPTB_tutorial.md

\section{Introduction}

Whole-genome sequencing (WGS) has become an integral part of basic and translational research on Mycobacterium tuberculosis, spanning topics ranging from pathogen evolution, epidemiology, and the emergence of drug resistance to TB latency and vaccine development ${ }^{1-6}$. Publicly accessible databases contain WGS data from thousands of clinical isolates of $M$. tuberculosis ${ }^{2,7,8}$. Phenotypic data such as drug susceptibility testing accompanies the WGS for several of these isolates, highlighting the usefulness of this data resource to the scientific community. In particular, identifying single nucleotide polymorphisms (SNPs) in WGS allows determining the functional significance of these mutations, such as their role in the emergence of drug resistance and adaptation to a new environment. In the absence of an open-source and stand-alone computational tool for easy analyses of WGS data, the data accumulation will continue to outpace extraction of meaningful information from sequencing data. 
SNPTB is a bioinformatics pipeline that combines widely used open-source software for WGS data quality control, read mapping to the M. tuberculosis reference genome, and SNP calling into a single python package. The input to the pipeline is the sequencing data in the fastq format (as is output by technologies such as Illumina) and it outputs high-confidence SNP calls. SNPTB has additional scripts for data organization and management as well as downstream analyses to identify the gene(s) (or the intergenic region) that harbor the mutations and to determine if the SNP(s) in a protein-coding gene is a synonymous or a nonsynonymous mutation. This pipeline is written for biologists with some exposure to the UNIX operating system.

\section{Implementation}

SNPTB is a python package for analyzing WGS data in the fastq format. The steps in the SNPTB pipeline are: i) data organization, ii) quality control, iii) read mapping, iv) SNP calling, and v) SNP annotation (Figure 1). In the data organization step, a python script creates a new folder for each sample (prefix in the fastq filenames serves as the sample name) and moves the associated fastq files into that folder. The subsequent quality control and read mapping steps create subfolders 'qc' and 'mapped' within each sample's directory.

During library preparation for Illumina sequencing, short sequences called 'adapters' are ligated to the DNA fragments. SNPTB uses open-source software Trimmomatic (version 0.36) to remove these adapter sequences from the reads output by the Illumina sequencers and to perform quality control analysis ${ }^{9}$. Reads shorter than 20 nucleotides are dropped. Trimmomatic also trims low-quality ends of the reads and clips a read if the average quality score in a window of four nucleotides falls below 20. FastQC then assess the quality of the filtered highquality reads ${ }^{10}$. The final filtered reads, the FastQC output, and the overview of the adapter trimming and quality control analyses are saved in the folder 'qc'.

The high-quality filtered reads are then mapped to the M. tuberculosis H37Rv reference genome (NCBI Accession AL123456.3) using the open-source read aligner software Bowtie 2 (version 2.2.6) (11 $^{13}$. The pipeline also outputs average depth (number of reads at each genome position) for each sample as well as the percent of the genome mapped by at least 5,10 or 20 reads. High confidence SNPs in the mapped reads (probability that a SNP call is incorrect $<1 \mathrm{e}-20$ ) are identified using open-source software SAMtools (version 1.2) and BCFtools (version 1.2) ${ }^{12,13}$. The SNP calls are made relative to the $M$. tuberculosis reference genome and are further annotated to determine the location of the SNP (gene name or intergenic region) as well as the functional consequence of the mutation (synonymous or nonsynonymous if the mutation lies in a protein-coding gene). The pipeline can also identify SNPs relative to another M. tuberculosis WGS sample instead of the reference genome. 


\section{Discussion}

While the cost of sequencing is decreasing, the high cost of computational analysis of WGS data and long turnaround time remains a challenge for biology laboratories that routinely generate appreciable amounts of sequencing data. Several opensource software for WGS data analyses exist, but they present a steep learning curve to bench biologists with limited computational experience. The goal of SNPTB is to facilitate bench biologist's access to computational software for SNP calling, which is one of the most common and straightforward analyses of WGS data. The python package comes with an example dataset and a step-by-step tutorial to utilize SNPTB for SNP calling and SNP annotation in M. tuberculosis genomes.

Data Organization

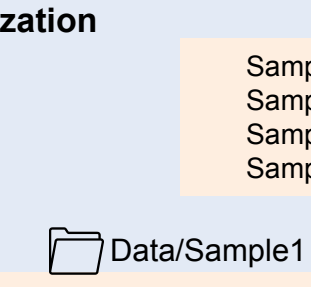

Sample1_R1_001.fastq.gz Sample1_R2_001.fastq.gz

\section{$\square$ Data}

Sample1_R1_001.fastq.gz

Sample1_R2_001.fastq.gz

Sample2_R1_001.fastq.gz

Sample2_R2_001.fastq.gz

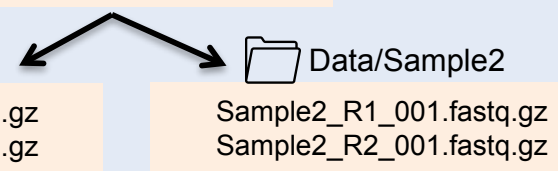

Figure 1. The SNPTB pipeline for WGS data analyses. Main steps in the pipeline are shown along with corresponding directory structure.

\section{$\sqrt{2}$}

Quality Control (Trimmomatic and FastQC)

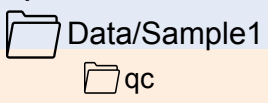

$\square \mathrm{qc}$

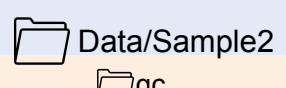

$\square \mathrm{qc}$

'qc' contains:

1. Filtered reads

2. FastQC output

3. Trimmomatic

run summary

\section{Read Mapping (Bowtie 2)}

$\square$ Data/Sample1

$\square$ qc
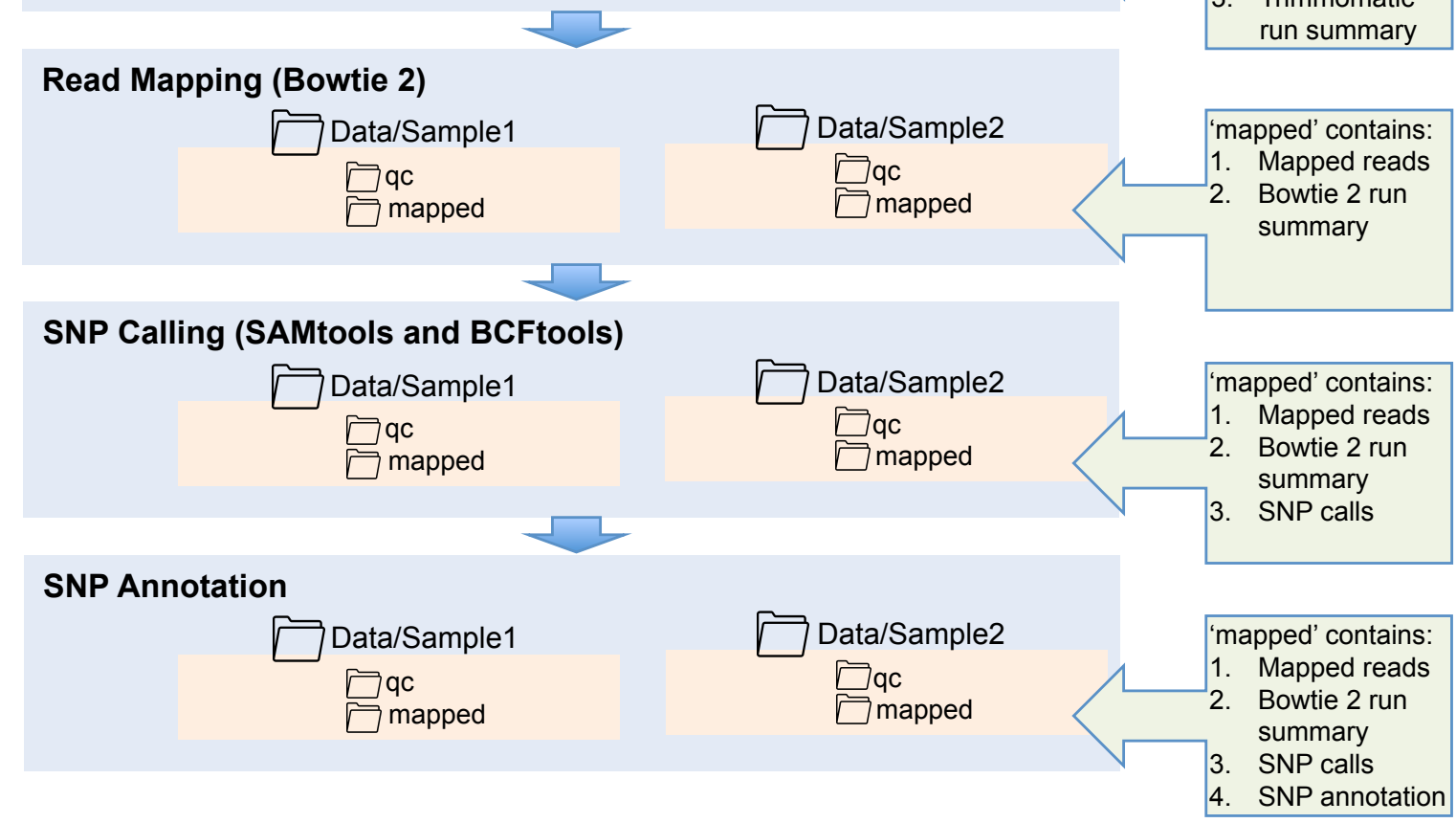


\section{Acknowledgements}

This work was supported by the NIH/NIAID Tuberculosis Research Unit grant U19Al11276. The funding source had no role in the study design; in the collection, analysis and interpretation of data; in the writing of the report; and in the decision to submit the article for publication.

\section{REFERENCES}

1. Lingaraju, S. et al. Geographic Differences in the Contribution of ubiA Mutations to High-Level Ethambutol Resistance in Mycobacterium tuberculosis. Antimicrobial Agents and Chemotherapy 60, 4101-4105 (2016).

2. Manson, A.L. et al. Genomic analysis of globally diverse Mycobacterium tuberculosis strains provides insights into the emergence and spread of multidrug resistance. Nature Genetics 49, 395-402 (2017).

3. Walker, T.M. et al. Whole-genome sequencing to delineate Mycobacterium tuberculosis outbreaks: a retrospective observational study. Lancet Infectious Diseases 13, 137-146 (2013).

4. Brosch, R. et al. Genome plasticity of BCG and impact on vaccine efficacy. Proceedings of the National Academy of Sciences of the United States of America 104, 5596-5601 (2007).

5. Ford, C.B. et al. Use of whole genome sequencing to estimate the mutation rate of Mycobacterium tuberculosis during latent infection. Nature Genetics 43, 482-+ (2011).

6. Colangeli, R. et al. Whole Genome Sequencing of Mycobacterium tuberculosis Reveals Slow Growth and Low Mutation Rates during Latent Infections in Humans. Plos One 9(2014).

7. Walker, T.M. et al. Whole-genome sequencing for prediction of Mycobacterium tuberculosis drug susceptibility and resistance: a retrospective cohort study. Lancet Infectious Diseases 15, 1193-1202 (2015).

8. Zhang, H.T. et al. Genome sequencing of 161 Mycobacterium tuberculosis isolates from China identifies genes and intergenic regions associated with drug resistance. Nature Genetics 45, 1255-U217 (2013).

9. Bolger, A.M., Lohse, M. \& Usadel, B. Trimmomatic: a flexible trimmer for Illumina sequence data. Bioinformatics 30, 2114-2120 (2014).

10. S, A. FastQC : a quality control tool for high throughput sequence data. (2010).

11. Langmead, B. \& Salzberg, S.L. Fast gapped-read alignment with Bowtie 2. Nature Methods 9, 357-U54 (2012).

12. $\mathrm{Li}, \mathrm{H}$. A statistical framework for SNP calling, mutation discovery, association mapping and population genetical parameter estimation from sequencing data. Bioinformatics 27, 2987-2993 (2011).

13. Narasimhan, V. et al. BCFtools/RoH: a hidden Markov model approach for detecting autozygosity from next-generation sequencing data. Bioinformatics 32, 1749-1751 (2016). 